\title{
Large-order behavior of the supersymmetric anharmonic oscillator
}

\author{
J. J. M. Verbaarschot \\ Department of Physics, State University of New York at Stony Brook, Stony Brook, New York 11794 \\ P. West \\ Department of Physics, University of Geneva, CH-1211 Geneva 4, Switzerland \\ and Department of Mathematics, King's College, Strand, London WC2R 2LS, United Kingdom* \\ Tai Tsun Wu \\ Theory Division, CERN, CH-1211 Geneva 23, Switzerland \\ and Gordon McKay Laboratory, Harvard University, Cambridge, Massachusetts $02138^{*}$
}

(Received 15 March 1990)

\begin{abstract}
The large-order behavior of two supersymmetric extensions of the anharmonic oscillator with two supersymmetries is found by explicit calculations. This behavior is also studied for deviations of the model away from supersymmetry. The supersymmetric point is a dividing point in that the behavior at the supersymmetric point and away from it in one direction is of the form $a_{0} a^{n} \Gamma(n+\alpha)$, while in the opposite direction it is of the form $b_{0} a^{n} \Gamma(n+\beta) \sin [(n+\gamma) 2 \pi / \tau]$.
\end{abstract}

\section{INTRODUCTION}

A long time ago, Dyson ${ }^{1}$ found that the perturbation series in QED is either divergent or convergent to an answer different from the exact one. In order to determine which one of the two possibilities holds, the anharmonic oscillator was studied in detail. ${ }^{2,3}$ In this case, it was found that the perturbation series for the energy levels are divergent. However, the series were shown to be Borel resummable. It was also found that all the energy levels of the even states are given by the different branches of one analytic function, while those of the odd states are given in the same way by a second analytic function. For the ground state, the coefficient of $g^{n}$ for large $n$ behaves asymptotically as $\left(6 / \pi^{3}\right)^{1 / 2}(-1)^{n+1} 3^{n} \Gamma\left(n+\frac{1}{2}\right)$. This result was obtained first by numerically fitting to an explicit computation, ${ }^{2}$ and later derived by a WKB analysis. ${ }^{3}$

The asymptotic behavior has also been reproduced by instanton techniques. ${ }^{4-7}$ The latter method has been generalized to field theories in two, three, and four dimensions, and requires a number of maneuvers of a delicate nature.

It is now believed that the asymptotic behavior of the anharmonic oscillator is quite general for a variety of field theories. ${ }^{7}$ The situation for QED, for which these questions were raised first, is still unknown. ${ }^{8-10}$

The above-mentioned issues lead to the well-known paradox: what is the theoretical basis for the most accurate measured number in physics, i.e., the $g-2$ of the electron? Of course we cannot exclude the possibility that the path integral of QED can be reconstructed from its perturbation series. However, as suggested by Dyson ${ }^{1}$ it could also be that QED must be embedded in another theory before one can give a meaning to its perturbation theory. We note that there are also other reasons for thinking that QED by itself is not a satisfactory theory.
For other theories of interest such as QCD the largeorder behavior is also unknown; this is one of the difficulties arising from our inability to estimate with certainty the large-order behavior in the presence of fermions.

Borel resummation has also been used to calculate with some accuracy the critical exponents of a number of models in statistical mechanics, including Ising-like models in two and three dimensions. ${ }^{11}$ The result of the present paper arose from an attempt by one of us (P.W.) to calculate the critical exponents in a supersymmetric Ising model. In the course of this calculation it became apparent that one required the large-order behavior of supersymmetric theories.

General theorems in supersymmetric theories guarantee that the ground-state vanishes at every order in perturbation theory. Thus at least for this level the usual divergent results at large order are not found. Indeed, this result had been quoted previously for certain potentials $^{12}$ which are, we now realize, supersymmetric. Furthermore, it implies that the analytic property connecting all energy levels as stated above must be modified for supersymmetric theories. For the first excited state there are no such theorems. It is thus natural to inquire whether the divergence and Borel summability are valid or not for the excited levels of a supersymmetric theory. The investigation of this question will be the subject of this paper.

As a starting point we study a supersymmetric extension of the harmonic oscillator. The potential is of the form

$$
V=\frac{1}{2} W^{\prime 2}-\frac{1}{2} W^{\prime \prime}
$$

where we have studied the cases $W_{1}^{\prime}=x+g x^{2}$ and $W_{2}^{\prime}=x+g x^{3}$. The most reliable way of finding the large-order behavior is to simply calculate numerically 
many orders using a computer and fitting the asymptotic behavior. For $W_{1}^{\prime}$ we found the result

$$
E_{2 n}=-\frac{18}{\pi} 3^{n}(n+1) !
$$

while for $W_{2}^{\prime}$ the result is

$$
E_{n}=\frac{8}{\pi}(-1)^{n+1} 2^{n} \frac{\Gamma\left(n+\frac{3}{2}\right)}{\Gamma\left(\frac{1}{2}\right)}
$$

for the parity-odd first excited state.

To discover whether the supersymmetric case is special, we also studied deviations about the supersymmetric point using the potentials

$$
V_{1}=\frac{1}{2} W_{1}^{\prime 2}-\frac{1}{2} W_{1}^{\prime \prime}+(d-1) g x^{3}-(c-1) g x,
$$

and

$$
V_{2}=\frac{1}{2} W_{2}^{\prime 2}-\frac{1}{2} W_{2}^{\prime \prime}+(d-1) g x^{4}-\frac{3}{2}(c-1) g x^{2} .
$$

For $c=d=1$ we recover the supersymmetric case. For $d=1, c \neq 1$ the asymptotic behavior for $V_{1}$ is given by

$$
E_{2 n}=-\frac{18}{\pi} 6^{1-c} 3^{n} \frac{\Gamma(n+3-c)}{\Gamma(2-c)},
$$

and for $V_{2}$ we find

$E_{2 n}=\frac{8}{\pi} 2^{3(1-c) / 4}(-1)^{n+1} 2^{n} \frac{\Gamma\left(n+\frac{3}{2}+\frac{3}{4}(1-c)\right)}{\Gamma\left(\frac{1}{2}+\frac{3}{4}(1-c)\right)}$.

However, for $d \neq 1$ and $c=1$ the behavior is very different. For $V_{1}$ with $d \geq 1$ the large-order behavior is of the form $a_{0} a^{n} \Gamma(n+\alpha)$ where now $a$ and $a_{0}$ are functions of $d$ but for $d<1$ the behavior is of the form

$$
a_{0} a^{n} \Gamma(n+\alpha) \sin (n+\gamma) \frac{2 \pi}{\tau},
$$

where $a_{0}, a, \gamma$, and $\tau$ are functions of $d$. This oscillatory behavior can also be expressed in terms of a complex multiplier $a$. For detailed analytical expressions we refer to Sec. VI. The results for $V_{2}$ are very similar. Consequently, the supersymmetric point is the dividing point between two regions of different types of asymptotic behavior.

\section{THE SUPERSYMMETRIC ANHARMONIC OSCILLATOR}

Supersymmetric quantum mechanics is the simplest supersymmetric system and has been a useful testing ground for ideas in supersymmetric theories. In particular, the breaking of supersymmetry has been investigated extensively in this model. ${ }^{13,14}$ In this paper we wish to investigate the behavior at large orders of perturbation theory in the hope that, as is the case for nonsupersymmetric models, it will be similar to that found in supersymmetric field theories.

We now recall the essential features of supersymmetric quantum mechanics with two supersymmetries. ${ }^{13}$ The model has one real even coordinate $x$ and two odd coordinates $\psi_{\alpha}, \alpha=1,2$. The action is

$$
S=\int d t\left(\frac{1}{2} \dot{x}^{2}-\frac{i}{2} \psi_{\alpha} \dot{\psi}_{\alpha}-\frac{1}{2} W^{\prime 2}-\frac{i}{2} W^{\prime \prime} \epsilon^{\alpha \beta} \psi_{\alpha} \psi_{\beta}\right),
$$

where $W$ is an arbitrary function of $x$ and

$$
\begin{aligned}
& W^{\prime} \equiv \frac{d W}{d x}, \quad W^{\prime \prime} \equiv \frac{d^{2} W}{d x^{2}}, \\
& \epsilon_{\alpha \beta}=\epsilon^{\alpha \beta}=-\epsilon^{\beta \alpha}, \quad \epsilon^{12} \equiv 1 .
\end{aligned}
$$

The Hamiltonian is given by

$$
H=\frac{1}{2} p^{2}+\frac{1}{2} W^{\prime 2}+\frac{i}{2} W^{\prime \prime} \epsilon^{\alpha \beta} \psi_{\alpha} \psi_{\beta} .
$$

Quantizing the usual way (i.e., $[x, \rho]=i \hbar,\left\{\psi_{\alpha}, \psi_{\beta}\right\}$ $\left.=\delta_{\alpha, \beta} \hbar\right)$ we find the Hamiltonian

$$
\hat{H}=-\frac{\hbar^{2}}{2} \frac{d^{2}}{d x^{2}}+V,
$$

where

$$
V=\frac{1}{2} W^{\prime 2}-\frac{\hbar}{2} \sigma_{3} W^{\prime \prime} .
$$

The energy levels of the system are given by the usual Schrödinger equation

$$
\hat{H} \psi=E \psi,
$$

where $\psi$ is a two-component spinor. As is characteristic for supersymmetric theories, the supercharges

$$
Q_{A}=\psi_{A} p-W^{\prime} \epsilon_{A B} \psi_{B}, \quad A=1,2
$$

generate the Hamiltonian by the relation

$$
\left\{Q_{A}, Q_{B}\right\}=2 \delta_{A B} H \text {. }
$$

The ground state is annihilated by the supercharges and so has $E=0$, and its wave function is given by

$$
\psi=\exp \left(W \sigma_{3} / \hbar\right)\left(\begin{array}{l}
0 \\
1
\end{array}\right),
$$

which can only be normalized when the highest power of $x$ in $W$ is even and has a positive coefficient. In the event that $\psi$ is not normalizable there is no supersymmetric ground state and supersymmetry is spontaneously broken. However, this fact does not show up in perturbation theory as a result of a general theorem for supersymmetric theories. Namely, if supersymmetry is not classically broken, then for these classically unbroken configurations the supersymmetric effective potential vanishes to all order of perturbation theory, and consequently supersymmetry will not be broken by quantum corrections at any order of perturbation theory..$^{15}$ In this case it means that all corrections to the ground state also vanish. ${ }^{15.16}$

We will be interested in generalizations of the anharmonic oscillator where the potential is $V=\frac{1}{2} x^{2}+g x^{4}$. In particular, we will consider the cases

$$
W_{1}^{\prime}=x+g x^{2},
$$


and

$$
W_{2}^{\prime}=x+g x^{3} .
$$

The wave function (2.9) is normalizable for the second case but not for the first case. Consequently, for $W_{1}$ there exists no supersymmetric nonperturbative ground state.

By a suitable scaling by $\hbar$ of $x, g$, and $E$ the Schrödinger equation for the above potentials reads, for the lowest component of $\psi$, which we also call $\psi$,

$$
\left(-\frac{d^{2}}{d x^{2}}+2 V\right) \psi=2 E \psi,
$$

where

$$
V=\frac{1}{2} W^{\prime 2}-\frac{1}{2} W^{\prime \prime}
$$

for the supersymmetric case. However, since we also will be interested in the large-order behavior for deviations from the supersymmetric case we will consider the two potentials

$$
\begin{aligned}
& 2 V_{1}=W_{1}^{\prime 2}-W_{1}^{\prime \prime}+2(d-1) g x^{3}-2 g(c-1) x, \\
& 2 V_{2}=W_{2}^{\prime 2}-W_{2}^{\prime \prime}+2(d-1) g x^{4}-3 g(c-1) x^{2},
\end{aligned}
$$

where the supersymmetric point is given by $c=d=1$. In what follows we will refer to the use of $V_{1}$ and $V_{2}$ as cases 1 and 2, respectively.

\section{DIFFERENCE EQUATIONS FOR THE ENERGY LEVELS}

Following Ref. 2 we will compute the perturbative corrections to the energy levels using difference equations which we will now derive. Since, as mentioned above, the ground-state energy vanishes at every order in perturbation theory, we will consider the first excited state. We consider the Schrödinger equation (2.12) with the potential (2.14). When we expand

$$
\begin{aligned}
& \psi=\sum_{n=0}^{\infty} P_{n}(x) g^{n} \exp \left(-x^{2} / 2\right), \\
& E=\sum_{n=0}^{\infty} E_{n} g^{n},
\end{aligned}
$$

and read off the coefficient of $g^{n}$, Eq. (2.12) becomes

$$
\begin{aligned}
-\frac{d^{2} P_{n}}{d x^{2}}+2 x \frac{d P_{n}}{d x}-2 E_{0} P_{n}-2 x & \left(c-d x^{2}\right) P_{n-1}+x^{4} P_{n-2} \\
= & 2 \sum_{p=1}^{n} E_{p} P_{n-p}
\end{aligned}
$$

By induction it follows immediately that the odd terms in the expansion (3.2) vanish (i.e., $E_{2 n+1}=0$ ), and that $P_{2 n}$ is an odd function of $x$ while $P_{2 n+1}$ is an even function of $x$. This results in the expansions

$$
\begin{aligned}
& P_{2 n}(x)=\sum_{q=0}^{3 n} x^{2 q+1} B_{2 n, q}, \\
& P_{2 n+1}(x)=\sum_{q=1}^{3 n+2} x^{2 q} A_{2 n+1, q} .
\end{aligned}
$$

From the boundary conditions for the first excited state which are such that the lowest-order coefficients are given by

$$
E_{0}=1 \text { and } P_{0}(x)=x,
$$

it follows immediately that

$$
\begin{aligned}
& B_{2 n, 3 n+1}=B_{2 n, 3 n+2}=\cdots=0, \\
& A_{2 n+1,3 n+3}=A_{2 n+1,3 n+4}=\cdots=0 .
\end{aligned}
$$

It also follows that all coefficients with negative indices are zero while

$$
B_{0,0}=1, \quad B_{2 n, 0}=0 \text { for } n \geq 1 \text {. }
$$

Substituting Eqs. (3.4) and (3.5) into Eq. (3.3) and comparing the coefficient of $x^{p}$ we find the difference equations

$$
\begin{aligned}
4 q B_{2 n, q}= & 2(2 q+3)(q+1) B_{2 n, q+1}-B_{2 n-2,, q-2} \\
& +2 c A_{2 n-1, q}-2 d A_{2 n-1, q-1} \\
& +2 \sum_{p=1}^{n} E_{2 p} B_{2 n-2 p, q}
\end{aligned}
$$

and

$$
\begin{aligned}
2(2 q-1) A_{2 n+1, q}= & 2(q+1)(2 q+1) A_{2 n+1, q+1} \\
& -A_{2 n-1, q-2}+2 c B_{2 n, q-1} \\
& -2 d B_{2 n, q-2}+2 \sum_{p=1}^{n} E_{2 p} A_{2 n+1-2 p, q} .
\end{aligned}
$$

Taking $q=0$ in Eq. (3.10) and using Eq. (3.9) we find that

$$
E_{2 n}=-c A_{2 n-1,0}-3 B_{2 n, 1} \text {. }
$$

Examining the boundary conditions Eqs. (3.7)-(3.9) and the difference Eqs. (3.10) and (3.11) we realize that starting at an odd level, say $2 n+1$, and taking $q=3 n+2,3 n+1, \ldots, 0$ successively one can express all the coefficients $A_{2 n+1, q}$ in terms of the coefficients with a smaller value for the first index. Similarly at level $2 n+1$ we start at $q=3 n+3,2 n+2, \ldots, 1$ and determine all the $B_{2 n+2, q}$ in terms of the previously found coefficients. As we will see in the next section, these equations are well suited to be analyzed numerically.

The perturbative expansion for the potential (2.15) is obtained in a similar way. Again we substitute the expansions for the wave function and the energy

$$
\begin{aligned}
& \psi=\sum_{n} B_{n}(x) g^{n} \exp \left(-x^{2} / 2\right), \\
& E=\sum_{p} g^{p} E_{p},
\end{aligned}
$$

in the Schrödinger equation (2.12). Since the potential $V_{2}$ is invariant under parity we have a parity-even and a parity-odd first excited state. For the parity-odd case we can write

$$
B_{n}(x)=\sum_{q=0} x^{2 q+1} B_{n, q}
$$


and the resulting difference equation is

$$
\begin{aligned}
2\left(2 q+1-E_{0}\right) B_{n, q}= & 2(q+1)(2 q+3) B_{n, q+1} \\
& -2 d B_{n-1, q-2}-B_{n-2, q-3} \\
& +3 c B_{n-1, q-1}+2 \sum_{p=1}^{n} E_{p} B_{n-p, q} .
\end{aligned}
$$

For the lowest parity-odd state we have $B_{0}(x)=x$ and $E_{0}=1$, and as a result we adopt the boundary conditions

$$
B_{0,0}=1, \quad B_{n, 0}=0, n \geq 1 .
$$

From the Schrödinger equation we find that the highest power of $x$ in $B_{n}(x)$ is equal to $2 n$. Therefore, we put

$$
B_{n, 2 n+1}=B_{n, 2 n+2}=\cdots=0 \text {. }
$$

Taking $q=0$ in Eq. (3.16) we find

$$
E_{n}=-3 B_{n, 1} \text {. }
$$

Similar results hold for the parity-even first excited state.

In the above potentials the supersymmetric case is obtained by taking $c=d=1$. In this case the difference equation that generates the perturbation series for an eigenvalue can be simplified. In the supersymmetric Schrödinger equation we put

$$
\psi=\bar{\psi} \exp \left(-\frac{W \sigma_{3}}{\hbar}\right),
$$

which results in the following equation for $\bar{\psi}$ :

$$
-\frac{\hbar^{2}}{2} \frac{d^{2} \bar{\psi}}{d x^{2}}+\hbar W^{\prime} \sigma_{3} \frac{d \bar{\psi}}{d x}=E \bar{\psi} .
$$

Absorbing $\hbar$ as described above we find, for the upper component of $\bar{\psi}$,

$$
-\frac{d^{2} \bar{\psi}}{d x^{2}}-2 W^{\prime} \frac{d \bar{\psi}}{d x}=2 E \bar{\psi} \text {. }
$$

One may worry that for certain cases $\exp \left(+W \sigma_{3} / \hbar\right)$ times a polynomial is not normalizable; however, in perturbation theory one is not sensitive to this difficulty, and the values for the energy levels obtained from this equation are identical to those obtained from Eq. (2.6).

To give an example, let us consider $W^{\prime}=x+g x^{3}$ and the first excited parity-even eigenstate. For this state we have

$$
E_{0}=2, \quad \bar{\psi}=x^{2}-\frac{1}{2} .
$$

Letting

$$
\bar{\psi}=\sum_{n=0}^{\infty} c_{n}(x) g^{n}, \quad E=\sum_{n=0}^{\infty} E_{n} g^{n},
$$

and

$$
c_{n}(x)=\sum_{q=1}^{n+1} x^{2 q} c_{n, q},
$$

the difference equation resulting from Eq. (3.22) is

$$
\begin{aligned}
2(q-1) c_{n, q}= & (q+1)(2 q+1) c_{n, q+1} \\
& -2(q-1) c_{n-1, q-1}+\sum_{p=1}^{n} c_{n-p, q} E_{p},
\end{aligned}
$$

where the boundary conditions for $c_{n, q}$ are

$$
\begin{aligned}
& c_{0,0}=1, \quad c_{0,1}=-\frac{1}{2}, \\
& c_{n, 0}=0, \quad n \geq 1, \quad c_{n, n+2}=c_{n, n+3}=\cdots=0 .
\end{aligned}
$$

All coefficients with negative indices are also zero. Taking $q=0$ in Eq. (3.26) we find

$$
E_{n}=2 c_{n, 1} \text {. }
$$

\section{LARGE-ORDER BEHAVIOR OF THE SUPERSYMMETRIC ANHARMONIC OSCILLATOR}

In this section we present the numerical results for the solutions of the difference equations given in the preceding section and obtain the asymptotic $n$ dependence of the coefficient $E_{2 n}$. Assuming that $E_{2 n}$ for the potential $V_{1}$ has a large $n$ dependence of the form $a_{0} a^{n} \Gamma(n+\alpha)$ we can compute $a$ and $\alpha$ from the largest coefficients that have been calculated. With these constants we compute a sequence $\hat{E}_{2 n}$ as

$$
\hat{E}_{2 n}=\frac{E_{2 n}}{A a^{n} \Gamma(n+\alpha)}
$$

and examine its convergence (the constant $A$ has been inserted for numerical convenience). Following Ref. 2 we fit $\widehat{E}_{2 n}$ to an expansion in $1 / n$,

$$
\hat{E}_{2 n}=a_{0}+\frac{a_{1}}{n}+\frac{a_{2}}{n^{2}}+\cdots+\frac{a_{\kappa}}{n^{\kappa}} .
$$

We evaluate the coefficients $a_{\kappa}$ from the last $\kappa+1$ energy levels $\hat{E}_{2 n}$ that have been calculated. The first coefficient is given by

$$
\left.a_{0}=\sum_{i=0}^{\kappa}(-1)^{i} \widehat{E}_{2\left(n_{\max }\right.}-i\right) \frac{\left(n_{\max }-i\right)^{\kappa}}{(\kappa-i) ! i !},
$$

where $E_{2 n_{\max }}$ is the coefficient for the maximum value of $n$ that has been computed. The coefficient $a_{1}$ is found by applying Eq. (4.3) to the last $\kappa-1$ terms $n\left(\widehat{E}_{2 n}-a_{0}\right)$ instead of the last $\kappa$ terms $E_{2 n}$. The remaining coefficients are found analogously.

When a sufficient long sequence of values $\hat{E}_{2 n}$ is available it is apparent from the monotonic convergence of $\widehat{E}_{2 n}$ whether the correct values for the multiplier $a$ and the number $\alpha$ have been found. This also follows from the behavior of the coefficients $a_{p}$ for increasing values of $\kappa$. Empirically we find that for $\kappa$ larger than an optimal value the coefficients start to diverge. In all cases this value was found to be $\kappa=6$. It is likely that the expansion (4.2) is an asymptotic expansion. Therefore, only the lowest coefficients $a_{p}$ can be determined accurately. The degree of accuracy can be estimated by comparing to 
coefficients for neighboring values of $\kappa$. If they agree in the first $q$ figures, we will say that the "expected accuracy" is one part in $10^{q}$. A high accuracy provides considerable confidence not only in the values of $a_{0}$ and $a_{1}$ but more importantly in the large-order behavior, i.e., $a^{n} \Gamma(n+\alpha)$. A further check of the accuracy is the agreement with the expectation that $a, \alpha$ and the $a_{p}$ can be expressed in terms of $\pi$, simple rational numbers and their roots.

The results for the supersymmetric models have been obtained from the first 245 nontrivial corrections to the energy level $E_{0}$. The coefficients $a_{p}$ quoted below have been derived from those with the optimal value of $\kappa$.

In the case $W^{\prime}=x+g x^{2}$ the large-order behavior of the first excited state (i.e., $E_{0}=1$ ) is given by

$$
\begin{gathered}
E_{2 n}=-(n+1) ! 3^{n}\left(\frac{18}{\pi}-\frac{143}{\pi} \frac{1}{n}+\frac{263}{\pi} \frac{1}{n^{2}}\right. \\
\left.+a_{3} \frac{1}{n^{3}}+\cdots\right),
\end{gathered}
$$

where $a_{3}$ is given approximately by 606.5. The coefficients $a_{0}, a_{1}$, and $a_{2}$ had an "expected accuracy" of 1 part in $10^{11}, 1$ part in $10^{7}$, and 2 parts in $10^{4}$, respectively. As an example we list in Table I some numerically obtained coefficients and their product with $\pi$. We see that $\pi a_{0}$ agrees with 18 to 1 part in $10^{12}$ while $\pi a_{1}$ and $\pi a_{2}$ agree with -143 and 263 to 1 part in $10^{11}$ and 1 part in $10^{4}$, respectively. We observe, as will generally be the case, that the coefficients agree much better with their identification than the expected accuracy would suggest.

In the case $W^{\prime}=x+g x^{3}$ we find the following largeorder behavior for the first excited parity odd (i.e., $\left.E_{0}=1\right)$ :

$$
\begin{aligned}
E_{n}= & (-1)^{n+1} \frac{\Gamma\left(n+\frac{3}{2}\right)}{\Gamma\left(\frac{1}{2}\right)} \frac{2^{n}}{\pi} \\
& \times\left(8-33 \frac{1}{n}+\frac{141}{16} \frac{1}{n^{2}}-103 \frac{1}{n^{3}}+\cdots\right),
\end{aligned}
$$

where the "expected accuracy" of $a_{0}, a_{1}$, and $a_{2}$ is 1 in $10^{11}, 1$ in $10^{9}$, and 1 in $10^{5}$, respectively. Their agreement with the above given numbers is 1 part in $10^{14}, 1$ part in $10^{10}$, and 1 part in $10^{7}$, respectively.

For the first excited parity-even state (i.e., $E_{0}=2$ ) of the same potential we find that

TABLE I. The coefficients $a_{k}$ and $\pi a_{k}$ of the expansion in $1 / n$ of $E_{2 n}$ as given in Eq. (4.2).

\begin{tabular}{lcc}
\hline \hline$k$ & $a_{k}$ & $\pi a_{k}$ \\
\hline 0 & 5.72957795130645 & 17.99999999999440 \\
1 & -45.518313719118 & -142.9999999837766 \\
2 & 83.72433563556 & 263.0277577593614 \\
3 & -193.04455148 & -606.4673447451046 \\
\hline
\end{tabular}

$$
\begin{aligned}
E_{n}= & (-1)^{n+1}(n+2) ! \frac{2^{n}}{\pi} 16 \sqrt{2} \\
& \times\left(1-\frac{99}{8} \frac{1}{n}+\frac{169}{3} \frac{1}{n^{2}}+a_{3} \frac{1}{n^{3}}+\cdots\right),
\end{aligned}
$$

where $a_{3}$ is approximately -185.1 . The "expected accuracy" for $a_{0}, a_{1}$, and $a_{2}$ is 1 part in $10^{10}, 1$ part in $10^{8}$, and 1 part in $10^{5}$, respectively. Their agreement with the above numbers is 1 part in $10^{12}, 1$ part in $10^{10}$, and 1 part in $10^{4}$, respectively.

We observe that the series generated by $E_{n}$ of Eqs. (4.5) and (4.6) are Borel resummable while that of Eq. (4.4) is not. We know, however, that in the latter case the perturbation series is constructing a state which nonperturbatively does not exist while it does exist in the former case. This agrees with the folklore which states that a series which is not Borel resummable has a nonperturbative instability.

\section{LARGE-ORDER BEHAVIOR AWAY FROM THE SUPERSYMMETRIC POINT}

In this section we study the anharmonic oscillator with the potentials $V_{1}$ and $V_{2}$ given in Eqs. (2.14) and (2.15), respectively. The supersymmetric point corresponds to $c=d=1$. The coefficient of the cross term $\frac{1}{2} W^{\prime 2}$ is varied by choosing $d-1 \neq 0, c=1$. For $d=1, c-1 \neq 0$ the coefficient of $-\frac{1}{2} W^{\prime \prime}$ is varied. In view of the origin of this term we may think of this as varying a Yukawa term. However, it is not completely clear whether in such a simplified model one can identify this term with what might, in a higher-dimensional analog, be the effect of adding fermions.

The large-order behavior for $d=1$ and arbitrary values for $c$ is not very different from the supersymmetric case. For the potential $V_{1}$ we find, for the coefficients of the first excited state,

$$
E_{2 n}=-3^{n} \frac{18}{\pi} 6^{1-c} \frac{\Gamma(n+3-c)}{\Gamma(2-c)} .
$$

Having identified the fact that the multiplier $a$ remains unchanged and that the factorial is $\Gamma(n+\alpha)$ with $\alpha=3-c$ we have applied the expansion (4.2) to this case for many values of $c$. In the range $-0.5 \leq c \leq 1.95$ the value of $a_{0}$ agreed to 1 part in $10^{8}$ or better with Eq. (5.1) (i.e., $a_{0}=18 / \pi 6^{1-c}$ ).

For $c=2$ the formula (5.1) yields $E_{n}=0$ due to the factor $\Gamma(0)$ in the denominator. This behavior could only be observed numerically by choosing $c$ slightly (say $10^{-20}$ ) different from 2. Since the initial rounding off error is multiplied each step by a large factor the calculated numbers for $E_{2 n}$ actually become very large instead of remaining zero. However, a careful numerical analysis shows that we have $E_{2 n}=0$ for all $n$ at $c=2$. Indeed, at $c=2 V_{1}$ is again a potential resulting from a supersymmetric model with $\bar{W}^{\prime}=W_{1}^{\prime}-1 / x=x+g x^{2}-1 / x$. The ground-state wave function for $\bar{W}$ is $\exp (-\bar{W})$ and so is of the form $x \exp \left(-x^{2}\right)+\cdots$ which starts in the same way as for the first excited state of the potential $W_{1}^{\prime}$.

Examining $V_{1}$ in the neighborhood of $c=3$ and $c=4$ 
one finds that although the coefficients $E_{2 n}$ do not vanish for small $n$, the coefficient $a_{0}$ vanishes and thus agrees with the above formula.

By varying $c$ for $d=1$ for the potential $V_{2}$ we find a similar large-order behavior. The coefficients $E_{n}$ for the first excited parity-odd state are given by

$E_{n}=\frac{8}{\pi} 2^{3(1-c) / 4}(-1)^{n+1} 2^{n} \frac{\Gamma\left(n+\frac{3}{2}+\frac{3}{4}(1-c)\right)}{\Gamma\left(\frac{1}{2}+\frac{3}{4}(1-c)\right)}$.

For $9 \geq c \geq-1$ the agreement of $a_{0}$ with the above is 1 part in $10^{8}$ or better. For $25 \geq c \geq 9$ the agreement was less impressive but convincing, and for $c \geq 30$ the convergence cannot be clearly established with the number of terms computed (i.e., 245).

For $c=\frac{5}{3}$ the above formula predicts that $E_{n}$ vanishes for large $n$, and in fact we find numerically that $E_{n}$ vanishes for all $n \geq 1$. It was also verified that Eq. (5.2) holds well for $c$ close to $\frac{5}{3}$. This phenomenon, like that for $V_{1}$, is a result of the fact that, for $c=\frac{5}{3}, V_{2}$ is a potential resulting from a supersymmetric model. What we have computed is the ground-state energy of the model with $\bar{W}^{\prime}=x+g x^{3}-1 / x$. At several other points where the $\Gamma$ function in the denominator becomes infinite (such as $c=11$ ) it was verified that $a_{0}=0$ in these cases. However, unlike at $c=\frac{5}{3}$ at these points $E_{n}$ does not vanish for all $n$ but, instead, only vanishes asymptotically. Again, for values of $c$ in the neighborhood of $c=11$, the formula (5.2) holds well.

In the last part of this section we study the case $c=1$ and $d \geq 1$. The behavior for $d<1$ is different and will be considered in the next section. We will largely restrict our attention to the potential $V_{1}$.

When we change $d$, unlike for $c$, the multiplier $a$ also changes, i.e., $a(d)$. This means that for each value of $d$ one must repeat the entire analysis from the beginning. Consequently, our numerical results in this range are less complete than those discussed previously and restricted to an understanding of the parametric dependence of the multiplier. We find that the terms $E_{2 n}$ are all negative and examining several cases it would seem that for $d>1$ the large- $n$ behavior is of the form $a_{0}(d) \Gamma(n+\alpha(d)) a^{n}(d)$.

Although the numerical analysis of the variation of $d \neq 1$ is more involved the analytical understanding of the results greatly simplifies. By rewriting the potential $V_{1}$ as

$$
\begin{aligned}
2 V_{1}=\frac{1}{g^{2}}\left\{W_{1}^{\prime 2}(g x)+2(d-1)(g x)^{3}\right. \\
\left.-g^{2}\left[W_{1}^{\prime \prime}(g x)+2(c-1) g x\right]\right\},
\end{aligned}
$$

one observes that the last term in the potential is subleading in $g$. In the case $d=1$, when the minima of the leading part of the potential are degenerate, a small symmetry-breaking term can have important effects. However, for $d \neq 1$ one expects this term to affect only the constant $a_{0}$ in the asymptotic expansion. This is also suggested by the fermionic origin of this term. The leading part of the potential was studied previously by Brezin, Le Guillou, and Zinn-Justin. ${ }^{7,17}$ The inverse multiplier is simply equal to the action of the one instanton
TABLE II. The $d$ dependence of the multiplier $a(d)$ in the asymptotic behavior of the coefficients $E_{n}$. We compare our numerical results (second column) to those obtained analytically for the leading part of the potential (third column).

\begin{tabular}{lcc}
\hline \hline \multicolumn{1}{c}{$d$} & $a(d)$ (numerical) & $a(d)$ (analytical) \\
\cline { 2 - 3 } 256 & 491454.8 & 491516.5 \\
128 & 122861.3 & 122876.9 \\
64 & 30712.93 & 30716.79 \\
32 & 7675.829 & 7676.785 \\
16 & 1916.549 & 1916.784 \\
8 & 476.7223 & 476.7793 \\
4 & 116.7461 & 116.7594 \\
2 & 26.66637 & 26.66918 \\
1.75 & 19.59290 & 19.59494 \\
1.50 & 13.42409 & 13.42547 \\
1.25 & 8.104751 & 8.105595 \\
1.2 & 7.127392 & 7.128144 \\
1.1 & 5.216144 & 5.216729 \\
1.05 & 4.236826 & 4.237347 \\
1.02 & 3.588299 & 3.588817 \\
1.01 & 3.337187 & 3.337760 \\
1.008 & 3.281724 & 3.282328 \\
1.005 & 3.192521 & 3.193221 \\
1.002 & 3.090347 & 3.091397 \\
1.001 & 3.049933 & 3.051371 \\
1.0005 & 3.026863 & 3.028621 \\
1.0003 & 3.016660 & 3.018497 \\
1.00025 & 3.013984 & 3.015811 \\
\hline \hline
\end{tabular}

solution:

$$
\frac{1}{a(d)}=-\frac{2}{3}+d^{2}-\frac{d}{2}\left(d^{2}-1\right) \ln \frac{d+1}{d-1} .
$$

For large $d$ the multiplier is proportional to $d^{2}$. This also follows by redefining $E_{n} \rightarrow d^{n} E_{n}$ and $P_{n} \rightarrow d^{n} P_{n}$ in Eq. (3.3). For $d$ very close to 1 the difference between the multiplier and 3 is proportional to $d-1$. In Table II we compare this value of $a(d)$ to the ones extracted from our numerical results and find an excellent agreement. It should be noted that the discrepancy is systematic. The value of $\alpha$, which is only determined in the next order $1 / n$, could not be obtained very accurately. Its value is consistent with $\frac{3}{2}$ which, according to Zinn-Justin, ${ }^{17}$ should be expected for the first excited state.

The region $c=1, d>1$ was also examined for the potential $V_{2}$, and a similar conclusion was reached. Namely, the asymptotic behavior is of the generic form

$$
a_{0}(-1)^{n+1} a^{n} \Gamma(n+\alpha),
$$

where $a_{0}$ and $a$ depend upon $d$. Also in this case analytical results for the leading part of the potential (after rescaling $x \rightarrow g^{1 / 2} x$ ) are available (see Ref. 7).

\section{AN OSCILLATORY LARGE-ORDER BEHAVIOR}

We now examine the potential $V_{1}$ [Eq. (2.14)] for $c=1$ and $d<1$. While the behavior for $d>1$ is of the standard type [i.e., $E_{n} \sim a_{0} \Gamma(n+\alpha) a^{n}$ ] that for $d<1$ shows a different behavior. This is immediately apparent from 
the appearance of successive blocks with negative and positive signs showing an oscillatory behavior with a period greater than 1 . The period $\tau$ of oscillations can be easily read off and for $d \rightarrow 1-$ they fit the formula

$$
\tau=\frac{2}{3} \frac{1}{1-d}
$$

The oscillatory behavior was also obtained analytically for the leading-order part of the potential. ${ }^{7}$ The reason is that for $d<1$ the potential has two complex zeros corresponding to two complex-conjugate saddle points of the Euclidean action $\mathcal{A}$. This results in an asymptotic behavior proportional to $\mathcal{A}^{n}+\mathcal{A}^{* n}$ which represents a sinusoidal dependence on $n$. The period $\tau$ is given by

$$
\frac{2 \pi}{\tau}=\arctan \frac{-\frac{\pi}{2} d\left(d^{2}-1\right)}{-\frac{2}{3}+d^{2}-\frac{1}{2} d\left(d^{2}-1\right) \ln \frac{1+d}{1-d}} .
$$

For $d \rightarrow 1_{\text {- }}$ this result converges to the asymptotic behavior given in Eq. (6.1). The inverse multiplier $a(d)$ is given by

$$
\frac{1}{a(d)}=\left|-\frac{2}{3}+d^{2}-\frac{1}{2} d\left(d^{2}-1\right)\left(\ln \frac{1+d}{1-d}+\pi i\right)\right| \text {. }
$$

We made a detailed study of one representative case where we choose $c=1$ and $d=0.95$. We assume that the large-order behavior is of the form

$$
E_{2 n} \sim a^{n} \Gamma(n+\alpha) f(n),
$$

where $f(n)$ is a periodic function with period $\tau$ (in this case $\tau \sim 18$ ). The constants $a$ and $\alpha$ can be obtained from the values of $E_{2 n}$ at the maxima of $f(n)$. We find $a=2.3219$ [Eq. (6.3) yields 2.3212] and $\alpha=1.5$ (again the value of $\alpha$ could not be determined accurately; we used the value that was obtained analytically ${ }^{17}$ for the leading part of the potential). From the sequence

$$
\hat{E}_{2 n}=\frac{E_{2 n}}{a^{n} \Gamma(n+\alpha)}
$$

it is apparent that the periodic function $f(n)$ is of the form

$$
f(n)=a_{0} \sin (n+\gamma) \frac{2 \pi}{\tau} .
$$

The values of $\gamma$ and $\tau$ are determined from the position of the most obvious zero and extremum of the $\hat{E}_{2 n}$ and fitting these to the above formula. We deduce that $\tau=18.2423$, and $\gamma=-226.45113$. The analytical result for $\tau$ is 18.2388. The value of $\gamma$ is equal to $1.57 \bmod (\pi / \tau)$, which differs significantly from the value of 1.5 that can be obtained ${ }^{17}$ for the leading part of the potential. The asymptotic $n$ dependence is thus given by

$$
E_{2 n} \sim \Gamma(n+\alpha) a^{n} \sin (n+\gamma) \frac{2 \pi}{\tau} .
$$

From the ratio of $E_{2 n}$ and its asymptotic dependence it is clear that we have found the correct asymptotic behavior for this case. We observe that the last 26 ratios are equal to $4.332(1+17.3 / n)$ up to 1 part in $10^{4}$. This also fixes the proportionality constant for Eq. (6.7). Its value is different from the constant obtained for the leading part of the potential.

Although we did not make a detailed study for other values of $d$, examination at several other values strongly suggests that the asymptotic behavior of the $E_{n}$ is of the form (6.7).

Also for the potential $V_{2}$ we find that for $c=1, d<1$ the asymptotic behavior is of the form $(-1)^{n+1} a^{n} a_{0} \Gamma(n+\alpha) f(n)$, where $f(n)$ is a periodic function with period $\tau$. For the $d$ dependence of the period we find

$$
\tau \rightarrow \frac{2}{1-d}
$$

for $d \rightarrow 1_{-}$in agreement with the analytical results for the leading part of the potential.

A similar detailed study of the case $c=1, d=0.9$ resulted in the asymptotic behavior of the form

$$
E_{n} \sim \Gamma(n+\alpha) a^{n} \sin (n+\gamma) \frac{2 \pi}{\tau}
$$

For $\gamma=-321.791832, \quad \tau=25.35824, \quad \alpha=1.5, \quad$ and $a=1.643595$ the ratio of $E_{n}$ and its asymptotic form is constant to one part in $10^{4}$ for the last 90 values of $E_{n}$ that have been calculated. Since $\alpha$ induces a $1 / n$ correction, its value is determined with an accuracy of only $\approx 5 \%$. The other constants are determined by a fit with an absolute accuracy of $\approx 10^{-5}$. The value of $\alpha$ agrees with what one expects ${ }^{17}$ for the first excited state for a potential with nondegenerate minima. The values of $a(d)$ and $\tau$ that can be deduced from the work of Brezin et $a l .^{7}$ are equal to 1.643537 and 25.357344 , respectively. An examination of the raw data for other values of $d<1$ strongly suggests that the asymptotic behavior is also of the above type but with different parameters.

We can conclude that both for $V_{1}$ and $V_{2}$ the asymptotic behavior for $d<1$ is of the form (6.7), and for $d \geq 1$ it is of the form $a_{0} a^{n} n$ !. We note that these two different asymptotic forms are continuously related. For $d \rightarrow 1$ the period $\tau \rightarrow \infty$ yielding a monotonous $n$ dependence for the coefficients $E_{n}$. However, the form of the behavior is not differentiable at $d=1$ as the periodic behavior does not reappear when $d>1$. In this sense the supersymmetric point is special in that it is a dividing point. The reason is that at this point the two zeros of the leading part of the potential become degenerate.

One may speculate that the behavior given in Eq. (6.7) is of a universal type for the departure from the supersymmetric point in a certain direction. At least for supersymmetric potentials derived from a polynomial function $W(x)$ we have that at the supersymmetric point the zeros of the leading part of the potential are coalescent. Generally speaking, when we change a parameter of this potential, in one direction we will obtain two real zeros whereas in the other direction we will obtain two complex zeros corresponding to an oscillatory behavior. In 
other words, there is at least one direction in which an infinitesimal deformation away from the supersymmetric point gives rise to Borel resummable asymptotic behavior.

It can be easily seen that an oscillatory series is Borel resummable. The Borel transformed series, i.e., the series obtained by dividing the coefficients by $\Gamma(n+\alpha)$, is of the form

$$
(g a t)^{n} \sin (n+\gamma) \frac{2 \pi}{\tau},
$$

where $t$ is the variable that enters in the Borel transformation. The series is convergent for $\mid$ gat $\mid<1$ and we may sum the series to the form

$$
\frac{1}{2 i} \frac{\exp \left(i(\gamma-1) \frac{2 \pi}{\tau}\right)}{\exp \left(-\frac{2 \pi}{\tau}\right)-g a t}-\frac{1}{2 i} \frac{\exp \left(-i(\gamma-1) \frac{2 \pi}{\tau}\right)}{\exp \left(+\frac{2 \pi}{\tau}\right)-g a t} .
$$

Thus the Borel summed function has poles at gat $=\exp ( \pm 2 \pi / \tau)$ which are not on the real axis except for $\tau \neq \infty$. Hence, in the naive sense the asymptotic behavior (6.7) is resummable for $\tau>0$.

\section{CONCLUSIONS}

We have examined the large-order behavior for the supersymmetric anharmonic oscillator and for deviations away from this point. It was found that the supersymmetric point is a dividing point in that at this point $d=1$ and varying $d>1$ the behavior is of the form $a_{0} a^{n} \Gamma(n+\alpha)$ while for $d<1$ it is of the form

$$
a_{0} a^{n} \Gamma(n+\alpha) \sin (n+\gamma)(2 \pi / \tau),
$$

where $a_{0}, \alpha, a, \gamma$, and $\tau$ depend on $d$ [see Eq. (6.7)]. In the latter region the asymptotic series is Borel resummable.

The simplified model of supersymmetric quantum mechanics can be thought of as the analog of the addition of fermions in a field theory such that the equation of motion for the fermionic variables is the "square root" of the equation of motion for the bosonic variables. As a result the bosonic part of the potential can be naturally ex- pressed as the square of another function that is usually assumed to be regular. Consequently, the zeros of this part of the potential are doubly degenerate. Then there will be a direction in which variation of the potential gives rise to complex zeros resulting in an oscillatory larger-order behavior. In this sense the supersymmetric point is special in the sense that it is a dividing point of some kind.

It would be interesting to recover completely the large-order behavior found in this paper using WKB or instanton techniques. These will be reported elsewhere. Having recovered these results one can proceed to the case of field theory. It would seem reasonable to conjecture that for the Wess-Zumino model with coupling $g$ the large-order behavior is of the form $g^{2 n}(-1)^{n} a^{n} \Gamma(n+\alpha)$. In four dimensions the four-loop beta function is known ${ }^{18,19}$ and would seem compatible with such a behavior.

A natural next step is the investigation of a model with a higher degree of supersymmetry. Along these lines we studied an $N=4$ model of supersymmetry. ${ }^{20}$ The results which show an almost identical pattern of behavior will be published as a Letter.

One could hope that the perturbation series of the theories of interest, i.e., QED, QCD, etc., can be interpreted in the framework of some kind of supersymmetric generalization. Much more work is required to arrive at definite conclusions. However, the present work does suggest that having added fermions to a bosonic theory the supersymmetry can modify the large-order behavior and therefore may play a role in the paradox outlined in the Introduction.

\section{ACKNOWLEDGMENTS}

Most of the work was carried out when all three of us were visiting CERN. We thank John Ellis, Maurice Jacob, and many others of the theory division for their kind hospitality and the use of the CERN Cray computer. One of us (P.C.W.) acknowledges the University of Geneva for its hospitality. The work of J. J. M. V. was supported in part by the U.S. Department of Energy under Grant No. DE-FG02-88-ER-40388. The work of T.T.W. was supported in part by the U.S. Department of Energy under Grant No. DE-FG02-84-ER40158.
${ }^{*}$ Permanent address.

${ }^{1}$ F. J. Dyson, Phys. Rev. 85, 631 (1952).

${ }^{2}$ C. M. Bender and T. T. Wu, Phys. Rev. 184, 1232 (1969).

${ }^{3}$ C. M. Bender and T. T. Wu, Phys. Rev. D 7, 1620 (1973).

${ }^{4}$ L. N. Lipatov, Pis'ma Zh. Eksp. Teor. Fiz. 24, 179 (1976) [JETP Lett. 24, 157 (1976)].

${ }^{5}$ L. N. Lipatov, Pis'ma Zh. Eksp. Teor. Fiz. 25, 116 (1977) [JETP Lett. 25, 104 (1977)].

${ }^{6}$ E. Brezin, G. Parisi, and J. Zinn-Justin, Phys. Rev. D 16, 408 (1977); 16, 996 (1977).
${ }^{7}$ E. Brezin, J. C. LeGuillou, and J. Zinn-Justin, Phys. Rev. D 15, 1544 (1977); 15, 1558 (1977).

${ }^{8}$ R. Balian, C. Itzykson, G. Parisi, and J. B. Zuber, Phys. Rev. D 17, 1041 (1978).

${ }^{9}$ J. Zinn-Justin, Phys. Rep. 70, 109 (1981).

${ }^{10}$ For a review, see G. 't Hooft, in The Why's of Subnuclear Physics, proceedings of the International School, Erice, Italy, 1977, edited by A. Zichichi (Subnuclear Series Vol. 15) (Plenum, New York, 1979).

${ }^{11}$ J. C. Le Guillou and J. Zinn-Justin, Phys. Rev. B 21, 3976 
(1980).

${ }^{12}$ I. Herbst and B. Simon, Phys. Lett. 78B, 304 (1978).

${ }^{13}$ E. Witten, Nucl. Phys. B185, 513 (1981).

${ }^{14}$ E. Witten, Nucl. Phys. B202, 253 (1982).

${ }^{15}$ P. West, Nucl. Phys. B106, 219 (1976).

${ }^{16}$ B. Zumino, Nucl. Phys. B89, 535 (1975).

${ }^{17}$ J. Zinn-Justin, Phys. Rep. 70, 109 (1981).
${ }^{18}$ L. V. Avdeev, S. G. Gorishny, A. Yu. Kamenshchik, and S. A. Larin, Phys. Lett. 117B, 321 (1982).

${ }^{19}$ A. Sen and M. K. Sundaresan, Phys. Lett. 101B, 61 (1981).

${ }^{20}$ J. J. M. Verbaarschot, P. West, and T. T. Wu, Phys. Lett. B 240, 401 (1990). 